\title{
Modifications of laser correlation spectrometer for investigation of biological fluids
}

\author{
Elina Nepomnyashchaya*, Zoja Zabalueva, Elena Velichko, and Evgenii Aksenov
}

Peter the Great St. Petersburg Polytechnic University, Institute of Physics, Nanotechnology and Telecommunications, 29 Polytechnicheskaya Str., St. Petersburg, 195251 Russia

\begin{abstract}
We suggest three modifications of a laser correlation spectrometer for analysis of complex multicomponent biological solutions based on: (i) dynamic light scattering with special signal processing for analysis of polydisperse solutions, (ii) use of polarimetric technique for shape analysis, (iii) cross-correlation processing. The schemes of the devices based on polarization and cross-correlation analysis are described.
\end{abstract}

\section{Introduction}

In modern medical physics the problem of research of biological solutions is solved by using various techniques, such as mass spectrometry, electronic and atomic microscopy, fluorescent spectroscopy [1] and nuclear magnetic resonance. Laser correlation spectroscopy (LCS) is used to study sizes of nanoparticles in liquids, however its applications for the analysis of multicomponent environments, such as blood serum, are limited. LCS is based on registration and analysis of light scattered from nanoparticles. We offer a combination of several registration schemes, which will allow distinguishing molecules in biological liquids, such as saliva and blood serum.

\section{Experimental setup}

The scheme of the experimental setup is presented in Fig. 1. A single-mode laser module with a power up to $2.5 \mathrm{~mW}$ and $650 \mathrm{~nm}$ wavelength was used. To achieve focusing, a nonspherical short-focus lens allowing us to obtain a $50 \mu \mathrm{m}$ laser beam diameter was used, the calculated extent of caustic in the solution was $5 \mathrm{~mm}$. Scattered radiation was registered under angles $5^{\circ}-175^{\circ}$ by a single-mode optical fiber connected to a photoelectronic multiplier. For the $90^{\circ}$ scattering angle, the calculated length of dispersion was $4.8 \mathrm{~mm}$. The signal from the photoelectronic multiplier was digitized by an ADC module with frequencies of $100 \mathrm{kHz}-50 \mathrm{MHz}$ and processed by a computer. This scheme allowed characterization of spherical nanoparticles with a high accuracy $( \pm 0.5 \mathrm{~nm})$.

To analyse aspheric nanoparticles, the polarimetric technique was used [2]. The scattered radiation was registered in two perpendicular polarizations, translational and rotational diffusion coefficients were calculated. Rotational diffusion coefficient gave us information

Corresponding author: elina.nep@gmail.com 
about shapes of the nanoparticles, which cannot be obtained with conventional LCS. By analyzing the polarization and depolarization scattering components simultaneously, we were able to determine the degree of nonsphericity for the albumin protein at different $\mathrm{pH}$ of the medium, which allowed us to determine real dimensions of the nonspherical molecules.

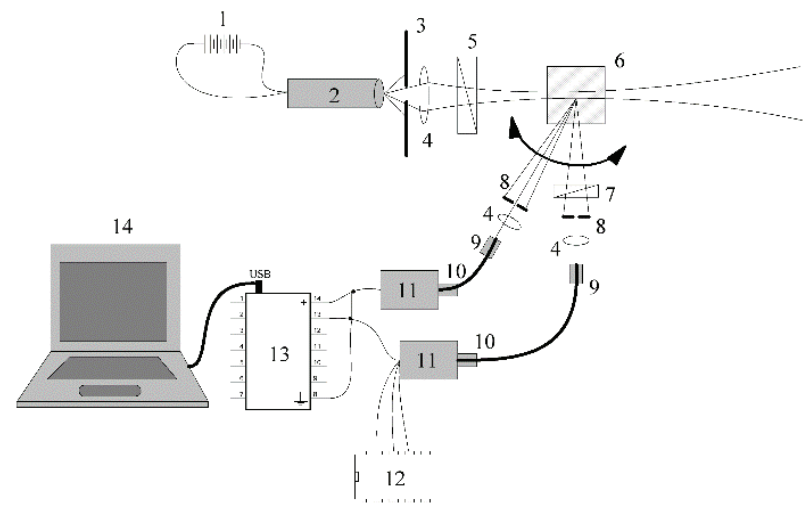

Fig. 1. Laser correlation spectrometer. 1 - rechargeable battery, 2 - laser, 3 - screen, 4 - lenses, 5 - polarizer, 6 - cuvette with the studied solution, 7 - output polarizer-analyzer, 8 - pinhole, 9,10 - input and output connectors of the single-mode fiber, 11 - photoelectronic multiplier, 12 stabilized power supply, 13 - ADC module, 14 - personal computer.

Our scheme gave us a possibility of a simultaneous registration of scattering at different angles and allowed us to exclude contributions of multiple scattering and to analyse solutions with high particle concentrations (non-Gaussian statistics) [3]. This allows studies of such liquids as blood and saliva in an undiluted form.

The signal registered in all schemes of measurements represents a dependence of the intensity of scattered radiation on time. To identify regularities in the scattered signals, the autocorrelation or cross-correlation (in the case of a simultaneous registration of two scattered signals) functions were calculated. Further analysis was carried out by using the original modification of the Tikhonov's mathematical technique for ill-posed problems [4].

Such biological liquids as blood serum and plasma, saliva, solutions of complement proteins, albumin, thrombin, various amino acids and also magnetic liquids were investigated [5]. Polydisperse size distributions of nanoparticles in the biological fluids were obtained. Polarization LCS allowed analysis of the nonsphericity of nanoparticles. Our crosscorrelation spectroscopic scheme will allow one to obtain these parameters for biological solutions in initial concentrations without the need for additional dilution and filtration, which can simplify the medical application of the device developed. The technique demonstrated a high efficiency in the analysis of biological solutions.

\section{References}

[1] S. Palmer, K. Litvinova, A. Dunaev, Biomed. Opt. Exp. 7, 1193 (2017)

[2] S.A. Dolgushin, I.K. Yudin, V.A. Deshabo, Biomed. Eng. 4, 394 (2016)

[3] C. Urban, P. Schurtenberger, Progr. Colloid Polim. Sci. 110, 61 (1998)

[4] E. Nepomnyashchaya, E. Velichko, E. Aksenov, J. Phys: Conf. Ser. 769, 012025 (2016)

[5] E. Nepomnyashchaya, E. Savchenko, E. Velichko, E. Aksenov, J. of Biomed. Phot. and Eng. 2, 040309 (2017) 